\title{
IMPLEMENTING TELEREHABILITATION RESEARCH FOR STROKE REHABILITATION WITH COMMUNITY DWELLING VETERANS: LESSONS LEARNED
}

\author{
NeAle R. Chumbler, PhD 1,2,3,4, PATricia Quigley, PhD, MPH, ARNP, CRN,
} FAAN 5 , JON SANFORD, M.ARCH ${ }^{6}$, PATRICIA GRIFFITHS, PHD ${ }^{6,7}$, DORIAN ROSE, PhD, PT 8 , Miriam MOREy, PHD ${ }^{9,10}$, E. Wesley Ely, MD, MPH ${ }^{11,12}$, HeLEN HOENIG, MD, MPH${ }^{13,14}$

${ }^{1}$ VA HSR\&D CENTER of EXCELLENCE ON IMPLEMENTING EVIDENCE BASED PRACTICE (CIEBP), RiCHARD L. ROUDEBUSH VAMC, INDIANAPOLIS, IN

${ }^{2}$ DEPARTMENT OF SOCIOLOGY, INDIANA UNIVERSITY SCHOOL OF LIBERAL ARTS, INDIANA UNIVERSITY

PURDUE UNIVERSITY INDIANAPOLIS, INDIANAPOLIS, IN

${ }^{3}$ VA HSR\&D STROKE QUALITY ENHANCEMENT RESEARCH INITIATIVE (QUERI) PROGRAM, RICHARD L. ROUDEBUSH VAMC, INDIANAPOLIS, IN

${ }^{4}$ REGENSTRIEF INSTITUTE, INDIANAPOLIS, IN

${ }^{5}$ Visn 8 PATIENT SAFETy Center, JAMES A. HALEy VAMC, TAMPA, FL

${ }^{6}$ ATLANTA VA REHAB R\&D CENTER, ATLANTA VAMC, ATLANTA GA

${ }^{7}$ EMORY UNIVERSITY SCHOOL OF MEDICINE, DiV. OF GERIATRICS AND GERONTOLOGY, ATLANTA, GA

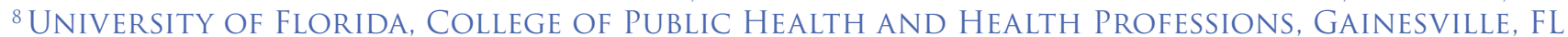

${ }^{9}$ DePARTMENT OF MEDICINE, DUKe UNIVERSITY MEDICAl CENTER, DURHAM, NC

${ }^{10}$ Geriatric Research EDUCATION AND Clinical CENTER, DURHAM VAMC, Durham, NC

${ }^{11}$ VANDERBILT UNIVERSITY MEDICAL CENTER, NASHVILLE, TN

12 Tennessee Valley HealthCare System, VA Geriatric Research Education and Clinical Center, NASHVILLE, TN

${ }^{13}$ Physical Medicine \& Rehabilitation Service, Durham VAMC, Durham, NC

${ }^{14}$ Department of Medicine, Duke University Medical Center, Durham, NC

\section{ABSTRACT}

Telerehabilitation (TR) is the use of telehealth technologies to provide distant support, rehabilitation services, and information exchange between people with disabilities and their clinical providers. This article discusses the barriers experienced when implementing a TR multi-site randomized controlled trial for stroke patients in their homes, and the lessons learned. The barriers are divided into two sections: those specific to TR and those pertinent to the conduct of tele-research. The TR specific barriers included the rapidly changing telecommunications and health care environment and inconsistent equipment functionality. The barriers applicable to tele-research included the need to meet regulations in diverse departments and rapidly changing research regulations. Lessons learned included the need for: telehealth equipment options to allow for functionality within a diverse telecommunications infrastructure; rigorous pilot testing of all equipment in authentic situations; and on-call and on-site biomedical engineering and/or IT staff.

\section{INTRODUCTION}

\section{ADVANTAGES OF TELEREHABILITATION}

Telehealth is the use of any communication modality (e.g., telephone, email, integrated video and audio, video teleconferencing, hand-held messaging), that enables physical separation of patient and practitioner while delivering health care services at a distance (Darkins
\& Cary, 2000). One primary goal of telehealth is to link patients with limited access and substantial needs to health care services (Nelson \& Palsbo, 2006). Among the various telehealth applications, telerehabilitation (TR) has received significant attention in recent years. TR is the use of telehealth technologies to provide distant support, assessment and information to people who have physical and/or neurological/cognitive impairments (Lai, Woo, Hui \& Chan, 2004; Russell, 2007; Schwamm et al., 2009). 
Effective implementation of a TR program can increase access to service and result in improved rehabilitation outcomes for individuals with physical impairments after discharge to home. First, TR increases patients' access to post-acute rehabilitation following hospitalization. TR can benefit people with disabilities residing in rural, remote locations because these individuals face incomplete service networks that threaten their safety and independent functioning (Demiris, Shigaki \& Schopp, 2005). Rural individuals also face more barriers accessing care because they travel farther to medical and rehabilitation appointments and have more transportation problems than their urban counterparts. Indeed, the farther rehabilitation programs are from rural residents' homes, the less likely residents are to receive services (Demiris, Shigaki \& Schopp, 2005; Johnson, Weinert \& Richardson, 1998). Second, TR can be provided at less cost than in-person services and can eliminate patients' travel time between their homes and the rehabilitation clinic (Dhurjaty, 2004; Kairy, Lehoux, Vincent \& Visintin, 2009). Third, TR reduces the need for therapists to travel to patients' homes while supporting real-time interactions between therapists and patients with physical disabilities in their home settings. The TR process ensures that treating therapists are aware of circumstances at home that may affect treatment. (Lai, Woo, Hui \& Chan, 2004). Fourth, an effective TR program can enhance continuity of care by enabling communication with the inpatient therapists who originally treated the patient after discharge home (Burdea, 2003; Sanford et al., 2006). Fifth, TR has great potential for improving both functional training and exercise training (Castro \& King, 2002; Green et al., 2002).

While TR holds great potential to improve access and outcomes, not many studies have implemented TR interventions, especially with community-dwelling stroke patients (Kairy, Lehoux, Vincent \& Visintin, 2009). A few studies have elucidated the barriers to and lessons learned from executing TR studies (Barrett, Larson, Carville \& Ellis, 2009; Demiris, Shigaki \& Schopp, 2005; Nelson \& Palsbo, 2006). Such barriers have included challenges in technology selection and operability; difficulties with human subjects compliance; telehealth devices that are too complicated for use by study participants; and insufficient staff training (Barrett, Larson, Carville \& Ellis, 2009; Demiris, Shigaki \& Schopp, 2005; Nelson \& Palsbo, 2006). The purpose of this paper is to present the lessons learned based on our implementation of a TR intervention for community dwelling stroke survivors. While our study enrolls only stroke patients, findings could have direct applicability to other patient populations when implementing TR studies. After briefly describing the context of the present study, we describe lessons learned specific to TR (i.e., the rapidly changing technology environment and problems related to functionality of the technology itself) and lessons pertinent to clinical research in general (i.e., interdepartmental coordination, and human subjects and information security protection challenges).

\section{DESCRIPTION OF TR INTERVENTION FOR STROKE PATIENTS}

The purpose of the TR intervention is to employ telehealth technology to improve outcomes of stroke patients after discharge to home. The primary aim is to determine the effect of TR on physical function, and secondarily to determine the effect on disability, fallsrelated self-efficacy (i.e., level of confidence possessed by stroke patients to not fall), and patient satisfaction. This study is a Phase II, 2-arm, 3-site randomized control trial (RCT). Subject enrollment began in 2009 and is currently ongoing. The study participants are recruited from three VA Medical Centers (VAMCs). Veterans who experienced an ischemic or hemorrhagic stroke within the preceding 24 months and who were discharged to the community are randomly assigned to one of two groups: (a) TR; and (b) usual care. Other inclusion criteria included age (4590 years); intact cognition; discharge motor Functional Independence Measure (FIM) score of 17-88 (Granger \& Hamilton, 1996); able to follow a 3-step command; agreement by the subject's physician; signed VHA Medical Media release document; and signed informed consent.

Study participants are identified via the automated Functional Status Outcome Database (FSOD) notification system located at each VAMC. The FSOD is a VHA administrative data source of stroke patients' disability and activity (i.e., Functional Independence Measure (FIM) (Granger \& Hamilton, 1996) evaluations of stroke patients. Specifically, the FSOD uses admission diagnoses and a variant of Reker's High Sensitivity ICD-9-CM algorithm (Reker et al., 2005) to notify clinicians that a patient with an acute stroke has been admitted to the hospital.

The TR intervention targets safe functional mobility of the individual within an accessible home environment via: 1) exercise (i.e., targeting underlying stroke-related impairment) and 2) adaptive strategies (i.e., targeting home safety and mobility factors to help compensate for physical disability). The TR intervention consists of three components: 1) three one-hour home visits by a trained assistant to assess physical performance and help communicate interventions recommended by the therapist; 2) daily participant use of an in-home messaging device that is monitored weekly by the therapist; and 3) five telephone calls between the therapist and the participant. The first home visit includes measures of static and dynamic sitting, standing balance and upper extremity range of motion. The battery includes the Performance-Oriented Motor AssessmentBalance (POMA-B) scale (Tinetti, 1986), a widely used tool for assessing mobility and falls risk in older people. Functional active range of motion of the upper extremities 
includes measures using a protocol developed by Jette and Branch (1984), and includes shoulder abduction/ external rotation, mass finger flexion and extension. No intervention is provided to the usual care group. However, both the TR and usual care groups received routine VA standard care and therapies ordered by their VA health care providers, and standard emergency contact numbers. Routine care may include home health nursing, nurse aide, and/or visits by rehabilitation practitioners.

The TR intervention is delivered over 3 months via home visits and telephone calls. Outcome assessments are conducted at baseline, 3-months and 6-months and administered remotely via telephone interviews with the following standardized instruments. The motor subscale of the FONEFIM (Smith, Hamilton \& Granger, 1990) is the primary outcome measure. There are four different instruments used to assess each of the four secondary research questions. The Late-Life Function and Disability Instrument (Salyers et al., 2004) is a two-part instrument used to assess disability (i.e., altered performance of major life tasks and social roles) and functional limitations (i.e., altered ability to perform specific activities encountered in daily actions) (Salyers et al., 2004). The Falls Self Efficacy Scale (Talley, Wyman \& Gross, 2008) is used to measure the fear of falling. Reker et al.'s (2002) Stroke-Specific Patient Satisfaction with Care is used to measure the participants' satisfaction.

\section{DESCRIPTION OF TECHNOLOGY}

In choosing a telehealth technology for our intervention, our choices were driven by these study criteria:

a. Technology will be used in a live two-way assessment and treatment of functional mobility tasks in diverse locations in the home.

b. Technology must function across the breadth of tele-infrastructure currently in the field (ranging from POTS only in rural areas, to wireless only, to DSL only). Excluding persons lacking high levels of tele-infrastructure would exclude most rural dwelling veterans, (a high priority for the VA and for telehealth) and would defeat the purpose of the program.

c. Technology must meet Health Insurance Portability and Accountability Act of 1996 (HIPAA) regulations.

Despite the wide array of available technologies, we were thus significantly limited in our choices, and ultimately chose to purchase two forms of technology to disseminate to TR study participants: 1) the TV 500 analog videophones from KMEA (San Diego, CA); and 2) the in-home messaging device (IHMD) (Health Buddy, Health Hero Network, Inc., Redwood City CA).

\section{IMPLEMENTATION BARRIERS}

The multi-site research team experienced two types of barriers, those that related to implementation of the TR, and those that related to implementation of the research. These barriers hindered the seamless implementation of the multi-faceted TR intervention. These barriers markedly lengthened the time needed to start recruitment, and in turn adversely affected sample size and study power, and required unanticipated changes in the study protocol. The TR specific barriers included rapidly changing technologies and problems with equipment functionality, whereas the general research related barriers included interdepartmental coordination and Human Subjects and Information Security Protection challenges.

Table 1. Barriers in Implementation of Telerehabilitation Intervention

\begin{tabular}{|c|c|}
\hline Barrier & Description \\
\hline \multicolumn{2}{|c|}{ 1. Changing Telecommunications and Health Care Environment } \\
\hline Obsolete Equipment & $\begin{array}{l}\text { Videophone operates on analog telephone } \\
\text { line as homes/offices are becoming digital }\end{array}$ \\
\hline Wireless Technology & Limited availability at some sites \\
\hline Home Health Agencies (HHA) & $\begin{array}{l}\text { Change in ownership and increase in price } \\
\text { made use of HHA Certified Nurse Assistants } \\
\text { (CNAs) not accessible and viable }\end{array}$ \\
\hline \multicolumn{2}{|l|}{ 2. Equipment Functionality } \\
\hline Videophone Limitations & $\begin{array}{l}\text { Resolution limited assessment to gross motor } \\
\text { skills }\end{array}$ \\
\hline Technical Support Needed & $\begin{array}{l}\text { Videophone function erratic; audio garbling; } \\
\text { home alarm systems }\end{array}$ \\
\hline \multicolumn{2}{|l|}{ 3. Inter-department Integration } \\
\hline Information Technology & $\begin{array}{l}4 \text { months to clarify classification of equipment } \\
\text { as IT }\end{array}$ \\
\hline $\begin{array}{l}\text { Clinical/IT/Research Funding } \\
\text { Mix }\end{array}$ & $\begin{array}{l}\text { 3-6 months across } 3-4 \text { different services to } \\
\text { hire hospital staff to work overtime on study }\end{array}$ \\
\hline Information Security & $\begin{array}{l}9 \text { months for wireless transmitter approval; } 6 \\
\text { months for approval to make a training video; } \\
\text { FSOD unavailability at one site }\end{array}$ \\
\hline \multicolumn{2}{|c|}{ 4. Changing Research and Human Subjects Protection Regulations } \\
\hline Human Subject Protection & $\begin{array}{l}10 \text { months to develop a training video due to } \\
\text { IT and Human Subject Protection concerns }\end{array}$ \\
\hline
\end{tabular}

\section{BARRIERS}

\section{BARRIER 1: RAPIDLY CHANGING}

TECHNOLOGIES.

The rapidly changing telecommunications and health care environments were manifest in a number of problems related to available technology. The videophones purchased for the study operated exclusively on analog telephone systems and quickly became obsolete. The grant to fund the study, which included the use of videophones, was originally submitted in late 2005 . The study received a fundable score in 2006, but was not awarded the funding to begin research until 2007. In order for this technology to be functional, both the teletherapist's office at the VAMC and the patient's home required analog lines. However, by the time the protocol 
was implemented, two of the three VAMC study sites had changed to entirely digital phone systems, so the local sites had to go through special procedures to have analog telephone lines installed. Simultaneously, many patients' homes transitioned from analog telephones to alternative methods of telecommunication (i.e., wireless only telephones); the analog based videophones were incompatible with DSL. When we pilot tested the videophones using DSL filters, the entire system failed, incapacitating the DSL and requiring several days to reinstate operability. Approximately 4 months after we purchased the videophones, the vendor discontinued both the product line and technical support.

To resolve the analog issue, the study team explored using Internet-based video-technology. However, Internet availability in the homes was highly variable (i.e., limited broadband/3G/T1 lines) and audio-video quality was problematic using wireless broadband. These experiences were consistent with a previous study by Nelson and Palsbo (2006), who reported that when they implemented video tele-clinics in physical therapy they had to broaden the study inclusion criteria over time due to technology and other problems, to increase recruitment and keep clinicians engaged. Nelson and Palsbo (2006) also found that technology was constantly evolving and presented significant limitations in their continued implementation. Ultimately, we resorted to videotaping the sessions for the therapists to review later and discuss with the patient via telephone.

\section{BARRIER 2: EQUIPMENT RELIABILITY.}

The second barrier centered on the operability of the telehealth equipment. The functionality of the analog videophone was erratic. When using the videophone and wireless camcorder/headset (linked by a wireless transmitter), sometimes audio was clear, sometimes not; sometimes the patient could be seen clearly, sometimes only their shadow could be seen; and sometimes the picture dropped out altogether. Audio problems were also experienced in the form of delays, garbling of words, and static, especially when the videophone was in close proximity to the wireless transmitter. Overall, the audio was better when the videophones were used rather than when the wireless camcorder/headphones were in use. We also experienced frequent problems with image quality, especially when the camcorder went from room to room. The in-home assistant frequently needed to adjust the videophone resolution and frequency (speed of transmission) for a better picture, requiring repeated attempts over several minutes to solve the problem, often to no avail. Also, not all of the in-home assistants were comfortable with technology and carrying out such adjustments, and on-call engineering help was not consistently available. DSL filters were employed to reduce the interference from DSL Internet/phone service, but this solution caused the system to terminate. Home alarm systems interfered with the videophones when the home alarm system was directly linked to the phone line, and any wireless technology in the home seemed to interfere (e.g., use of a wireless phone system or microwave oven).

We developed a variety of alternative procedures to deliver the intervention in the face of equipment problems, which in turn required submitting protocol amendments to the IRB and further delaying progress with the study. We found that having a biomedical engineer on contract with the study for real-time technical support enabled the barriers to be overcome more efficiently, but the original grant proposal did not include funding for local biomedical engineers at all of the study sites.

\section{BARRIER 3: INTERDEPARTMENTAL \\ COORDINATION.}

The third barrier refers to delays caused by efforts to secure organizational coordination between departments. Telerehabilitation studies within large healthcare or university-based institutions typically require the coordinated involvement of multiple departments, (e.g., research, information technology, fiscal, human resources, and various clinical services). In our experience, less than optimal interdepartmental coordination hindered and delayed study implementation in three main areas: 1) equipment purchase and the development of study materials; 2) implementation of study protocols; and, 3) the hiring of study personnel.

Approval was needed from multiple departments before the study equipment could be purchased. Specifically, agreements needed to be reached as to whether particular types of equipment (e.g., the wireless transmitter) were defined as IT equipment or medical equipment. These opinions differed across the study sites, and at the local and national levels for the respective services. That determination, in turn, affected the mechanism by which the equipment could be purchased (i.e., money in the grant versus money centrally appropriated to Information Resource Management Service). The net result was that it took 11 months to purchase and receive all of the study equipment.

\section{BARRIER 4: HUMAN SUBJECTS AND INFORMATION SECURITY PROTECTION.}

A fourth barrier experienced in implementing the study involved the intricate gradations with the protection of human subjects and information security for technology-based equipment (i.e., videophones, in-home communication devices), procedures and data transfer. The development of video-based training materials was complicated by information security and protection of human subjects requirements that were newly developed 
since the study was designed, which in turn caused problems obtaining access to needed technology and equipment. For example, the production of a TR training video took 10 months to complete due to the complexities of using an external non-VA facility to blur subject faces that ultimately involved approval by the local VA Information Security Officer and Privacy Officer, the local VA Institutional Review Board and the Institutional Review Board at the other facility. A Data Use Agreement was established, and a VA encrypted thumb drive had to be obtained through Information Technology Service to allow for transportation of the digital images between facilities.

\section{LESSONS LEARNED}

We developed a variety of solutions to meet the problems we encountered, with some solutions proving more successful than others. Table 2 depicts each barrier, followed by the solution for each barrier.

Table 2. Solutions to Barriers in Telerehabilitation Implementation

\begin{tabular}{ll}
\hline Barrier & Solution \\
\hline 1. Changing Telecommunications and & $\begin{array}{l}\text { Diverse telehealth equipment } \\
\text { Health Care Environment }\end{array}$ \\
& $\begin{array}{l}\text { available } \\
\text { Back-up strategies to meet } \\
\text { staffing needs }\end{array}$ \\
\hline 2. Equipment Functionality & Rigorous pilot testing \\
& - Bioengineering and/or IT \\
& support on-call and on-site \\
& Funding to replace/purchase \\
& new equipment \\
& Equipment failure back-up \\
& strategies written in protocol \\
\hline 3. Inter-department Integration & Early contact with relevant \\
& services at levels \\
\hline 4. Changing Research and Human & Dedicated and experienced \\
Subjects Protection Regulations & research coordinator at each \\
& site \\
\hline
\end{tabular}

The first barrier was the rapidly changing telecommunications and health care environment. Having varied telehealth equipment available to allow for functionality with the currently diverse telecommunications infrastructure would eliminate this problem. Our final protocol for the televideo portion of the study allowed for use of Internet-based or analogbased video communication or simple video-recording, with voice communication relying on analog, digital or cell phone technology. In addition, we found it helpful to have back-up strategies identified to meet staffing needs across the differing health care environments. Across our sites, we used a combination of contract services, hired part-time and full-time staff, and paid existing clinical staff via overtime salary.

The second barrier was the unreliability of telehealth equipment. We believe there are several solutions for this barrier, including rigorous pilot testing all equipment in diverse situations and with diverse personnel. It is also important to ensure that biomedical engineering or IT staff members are on-call and accessible at all sites. Equipment funding should be available in all years of the study to replace worn or defective equipment and to purchase new technologies that may become available. Back-up strategies should be incorporated in the protocol for equipment failures (e.g., videotaping study participants with telephonic communications).

A third barrier included the challenge of accommodating the regulations in diverse departments. We recommend that investigative teams work with relevant services at the local, regional, and national levels to anticipate the various regulations required to implement a telehealth study. This involvement can occur while the investigators are writing the grant and awaiting funding, so that purchases can be made quickly.

A fourth barrier was the rapidly expanding and changing human resources, information technology, and research regulations. One imperative solution we suggest is an experienced research coordinator at each study site with the dedicated time to assist study personnel through this complex system. A second solution is to advocate for greater standardization of human subjects (e.g., education and diagnosis), so that the research training of various study personnel can be more appropriately focused.

\section{DISCUSSION AND RECOMMENDATIONS}

This article presents four barriers that uniquely hindered an efficient implementation of a TR intervention and offers specific solutions. The barriers include the rapidly changing telecommunications and health care environment; the lack of consistent telehealth equipment functionality; discoordination between clinical, information technology, and research departments; and new regulations leading to more stringent protection for human subjects.

Despite the failure with the videophone implementation, we were able to effectively and appropriately implement the messaging device in individuals with land-line telephones. However, it is important to note that equipment-based challenges have also been reported in other telehealth studies. In one AHRQ project, one third of the patients had significant difficulties using the devices and therefore stopped using them (AHRQ highlights benefits, challenges of telehealth, 2009). In another study, heart failure patients who used an inhome telehealth communication device found it to be bothersome and complex to use (LaFramboise, Woster, Yager \& Yates 2009). The researchers asked a small sample $(n=13)$ of these patients about their perceptions of use, efficacy and difficulties encountered by using a home-telehealth messaging device. The patients found 
the device inflexible. They reported that the content on the device was monotonous and did not offer enough response options; the device could only be used where there was an available telephone jack and electrical outlet; and, they were bothered while sleeping at night by the screen's bright light. A recent telehealth RCT of hypertensive patients provided patients in the intervention arm with a home blood pressure (BP) telemonitoring device. McCant and colleagues (2009) reported that $61 \%$ of non- transmitted alerts regarding missed BP recordings, were not due to patient non-adherence, but rather due to equipment malfunction.

Based on our experience, we recommend that telehealth equipment be simplified in operation to create simple, reliable, high quality cost effective systems that can add different functions as needed. More research that uses a "bottom up" strategy is warranted wherein the patients' perspectives, needs, and expectations about telehealth devices are first consulted, followed by an iterative approach in which they are involved in decisions throughout development.

TR holds great potential for addressing health personnel shortages and reducing the costs of care delivery, especially for frail older individuals living in rural communities where access to rehabilitation care is severely limited. Specifically, TR for stroke patients is an innovative way to meet the needs of stroke survivors who may not otherwise have efficient access to timely healthcare. However, it is important to be cognizant of the needs and perceptions of the patients and how that interfaces with their post-discharge home environment (Lutz, Chumbler, Lyles, Hoffman \& Kobb, 2009). The findings suggest that the development, implementation and evaluation of stroke TR interventions are highly complex, and this complexity is frequently not reported or is underrated in many published telerehabilitation studies (May et al., 2002).

Our study highlights the need to address from the outset the challenges that arise from both technology use and the intricacies of interdepartmental planning; the challenges of rapidly changing technologies; and the need to pilot technology with adequate technical supports before deploying full scale research or clinical activities.

\section{ACKNOWLEDGMENT}

This research was supported by the Department of Veteran Affairs Rehabilitation Research and Development Service (B4492R). The opinions contained in this paper are those of the authors and do not necessarily reflect those of the US Department of Veterans Affairs. No potential conflicts of interest exist. 


\section{REFERENCES}

AHRQ highlights benefits, challenges of telehealth. (2009). Healthcare Benchmarks and Quality Improvements, 16, 16-18.

Barrett, M., Larson, A., Carville, K., \& Ellis, I. (2009). Challenges faced in implementation of a telehealth enabled chronic wound care system. Rural and Remote Health, 9, 1154 (Online).

Burdea, G.C. (2003). Virtual rehabilitation - benefits and challenges. Methods of Information in Medicine, 42,51923.

Castro, C. \& King, A.C. (2002). Telephone-assisted counseling for physical activity. Exercise and Sport Sciences Reviews, 30, 64-68.

Darkins, A.W. \& Cary, M.S. (2000). Telemedicine and Telehealth. Springer Publishing Company, 173-179.

Demiris, G., Shigaki, C.L., \& Schopp, L.H. (2005). An evaluation framework for a rural home-based telerehabilitation network. Journal of Medical Systems, 29,595-603.

Dhurjaty S. (2004). The economics of telerehabilitation. Telemedicine Journal \& E-Health, 10, 196-199.

Granger, C.V \& Hamilton, B.B. (1996). Measuring Health: A guide to rating scales and questionnaires. In $\mathrm{C}$. Newell \& I. MacDowell (Eds). Measuring health: A guide to rating scales and questionnaires, (2nd ed.). Oxford University Press, (pp. 115-121).

Green, B.B., McAfee, T., Hindmarsh, M., Madsen, M., Caplow, M., \& Buist. D. (2002). Effectiveness of telephone support in increasing physical activity levels in primary care patients. American Journal of Preventive Medicine, 22, 177-183.

Jette , A.M. \& Branch, L.G. (1984). Musculoskeletal impairment among the non-institutionalized aged. International Rehabilitation Medicine. 6, 157-161.

Johnson, J.E., Weinert, C., \& Richardson, J.K. (1998). Rural residents' use of cardiac rehabilitation programs. Public Health Nursing, 15, 288-296.

Kairy, D., Lehoux, P., Vincent, C., \& Visintin M. (2009). A systematic review of clinical outcomes, clinical process, healthcare utilization and costs associated with telerehabilitation. Disability and Rehabilitation, 31, 427-447.

LaFramboise, L.M., Woster, J., Yager, A., \& Yates, B.C. (2009). A technological life buoy: patient perceptions of the Health Buddy. Journal of Cardiovascular Nursing, 24, 216-24.

Lai, J.C.K, Woo, J., Hui, E., \& Chan, W.M. (2004). Telerehabilitation- a new model for community-based stroke rehabilitation. Journal of Telemedicine and Telecare, 10, 199-205.

Lutz, B.J., Chumbler, N.R., Lyles, T., Hoffman, N., \& Kobb, R. (2009). Testing a home-telehealth programme for US veterans recovering from stroke and their family caregivers. Disability and Rehabilitation, 31, 402-9.
May, C.R., Williams, T.L., Mair, F.S., Mort, M.M., Shaw, N.T., \& Gask, L. (2002). Factors influencing the evaluation of telehealth interventions: preliminary results from a qualitative study of evaluation projects in the UK. Journal of Telemedicine and Telecare, 8, 65-67.

McCant, F., McKoy, G., Grubber, J., Olsen, M.K., Oddone, E., et al. (2009). Feasibility of blood pressure telemonitoring in patients with poor blood pressure control. Journal of Telemedicine and Telecare, 15, 28185.

Nelson, E.L. \& Palsbo, S. (2006). Challenges in telemedicine equivalence studies. Evaluation and Program Planning, 29, 419-425.

Reker, D.M., Duncan, P., Horner, R.D., Hoenig, H., Samsa, G.P., Hamilton, B.B., \& Dudley, T.K. (2002). Postacute stroke guideline compliance is associated with greater patient satisfaction. Archives of Physical Medicine and Rehabilitation, 83, 750-756.

Reker, D.M., Reid, K., Duncan, P.W., Marshall, C., Couper, D., et al. (2005). Development of an integrated stroke outcomes database within Veteran Health Administration. Journal of Rehabilitation Research and Development, 42, 77-92.

Russell, T.G. (2007). Physical rehabilitation using telemedicine. Journal of Telemedicine and Telecare, 13, 217-220.

Salyers, S.P., Jette, A. M., Haley, S.M., Heeren, T.C., Guralnik, J.M., \& Fielding, R.A. (2004). Validation of the Late-Life Function Disability Instrument. Journal of the American Geriatrics Society, 52, 1554-1559.

Sanford, J.A., Griffiths, P.C., Richardson, P., Hargraves, K., Butterfield, T., \& Hoenig, H. (2006). The effects of in-home rehabilitation on task self-efficacy in mobilityimpaired adults: a randomized clinical trial. Journal of the American Geriatrics Society, 5, 1641-8.

Schwamm, LH., Holloway, R.G., Amarenco, P., Audebert, H.J., Bakas, T., et al. (2009). A review of the evidence for the use of telemedicine within stroke systems of care. A scientific statement from the American Heart Association/ American Stroke Association. Stroke, 40, 2616-34.

Smith, P., Hamilton, B.B., \& Granger, C.V. (1990). Functional independence measure decision tree. The FONE FIM. Buffalo, NY: Research Foundation, State University of New York.

Talley, K.M., Wyman, J.F., \& Gross, C.R. (2008). Psychometric properties of the activities specific balance confidence scale and the survey of activities and fear of falling in older women. Journal of the American Geriatrics Society, 56, 328-333.

Tinetti, M.E. (1986). Performance-oriented assessment of mobility problems in elderly patients. Journal of the American Geriatrics Society, 34, 119-126. 
